\title{
Exposure Start Time
}

National Cancer Institute

\section{Source}

National Cancer Institute. Exposure Start Time. NCI Thesaurus. Code C83133.

The time at which the exposure begins. 